\title{
Perturbed Integral Collocation Approximation Method For The Solution of a Class of Second Order Differential Equations
}

\author{
+Taiwo, O . A, Faniyi , O . E, Bello, K. A and *Okperhie, E. P \\ Department of Mathematics Faculty of Science, University of Ilorin, Nigeria \\ *Department of Mathematics and Statistics, Federal Polytechnic, Bida, Nigeria.
}

\begin{abstract}
This paper concerns the numerical approximation of a class of second order linear ordinary differential equations by Perturbed Integral Collocation Method.

The application of the method resulted to system of linear algebraic equations which are then solved by Gaussian elimination method. The results obtained for some illustrative examples showed that the Perturbed Integral Collocation Method is efficient, accurate and reliable.

Examples are given to illustrate the method.
\end{abstract}

Key words: Perturbed Integral Collocation, Polynomials and Chebyshev AMS Subject Classification: 65L10 + Author to communicate concerning the paper

\section{Introduction}

The demands for the use of numerical methods have been on the increase in recent years. One reason for this is that analytical methods can only provide solution for linear, quadratic, and cubic equations to mention a few. On the other hand, numerical methods provide solutions to all polynomial equations, transcendental, hyperbolic equations and others. In this work, numerical method based on Perturbed Integral Collocation Approximation Method is used to obtain numerical solutions of linear and nonlinear second order Ordinary Differential Equations.

Several research works have been carried out in these areas in recent years. Second order linear and nonlinear ordinary differential equations are solved numerical for various methods (see ref. [1, 2, 4, 5 ]. Other methods include Finite Difference Method (FDM), Adomian Decomposition Method (ADM), Homotopy Analysis Method (HAM) and Variational Iteration Method (VIM). [3] solved Ordinary Differential Equations using the Perturbation term of the Tau Method over Semi-Infinite Intervals. All the above numerical methods motivated the idea reported in this paper.

\section{Chebyshev Polynomials}

The Chebyshev polynomials of the first kind denoted by $T_{n}(x)$ is defined by

$T_{n}(x)=\operatorname{Cos}\left(n \operatorname{Cos}^{-1}(x)\right), \quad-1 \leq x \leq 1$

Where $\mathrm{n}$ is a non negative integers and the recurrence relation is given as

$T_{n+1}(x)=2 x T_{n}(x)-T_{n-1}(x), \quad n \geq 0$

\section{Error}

In this work, we have defined error as the difference between the exact and approximate solutions when evaluated at some equidistant points within the intervals of consideration. This is represented mathematically as

$$
\text { Error }=\left|y(x)-y_{N}(x)\right|
$$

Where $\mathrm{y}(\mathrm{x})$ is the exact solution, $\mathrm{y}_{N}(\mathrm{x})$ is the approximate solution and $\mathrm{N}$ is the degree of the approximation used.

\section{Problem Considered}

In this section, we considered the general form of linear second order ordinary differential equation given as $\left(p_{20}+p_{21} x+p_{22} x^{2}\right) y^{\prime \prime}(x)+\left(p_{10}+p_{11} x\right) y^{\prime}(x)+p_{00} y(x)=f(x), \quad a \leq x \leq b$ Where,

$p_{r k}, r, k=0(1) 2$ are constants and $f(x)$ is any given smooth function (Polynomials, transcendental functions or hyperbolic function ) together with the associated conditions 
$y(a)+y^{\prime}(a)=\alpha_{0}$

And,

$y(b)+y^{\prime}(b)=\alpha_{1}$

Description of Perturbed Integral Collocation Approximation Method

For the purpose of using Perturbed Integral Collocation Method to solve equations (2.1) - (2.3), we assumed an approximate solution of the form:

$$
y_{N}(x)=\sum_{r=0}^{N} a_{r} x^{r}
$$

Thus, equation (2.4) is substituted into a slightly perturbed equation (2.1) and we have,

$$
\begin{array}{ll}
\left(p_{20}+p_{21} x+p_{22} x^{2}\right) y_{N}{ }^{\prime \prime}(x)+\left(p_{10}+p_{11} x\right) y_{N}{ }^{\prime}(x)+p_{00} y_{N}(x) \\
=\sum_{r}^{N} f_{r} x^{r}+\tau_{1} T_{N}(x)+\tau_{2} T_{N-1}(x), & a \leq x \leq b
\end{array}
$$

Where,

$T_{N}(x)=\sum_{r=0}^{N} C_{r}^{(N)} x^{r}$

And ,

$T_{N-1}(x)=\sum_{r=0}^{N-1} C_{r}^{(N-1)} x^{r}$

Here, $a_{0}, a_{1}, a_{2}, \ldots \ldots \ldots \ldots, a_{N}, \tau_{1}$ and $\tau_{2}$ are to be determined.

Thus, we integrated equation (2.5) twice as follows:

$$
\begin{aligned}
\int_{0}^{x} \int_{0}^{u}\left(p_{20}+p_{21} t+p_{22} t^{2}\right) y_{N}{ }^{\prime \prime}(t) d t d u & +\int_{0}^{x} \int_{0}^{u}\left(p_{10}+p_{11} t\right) y_{N}{ }^{\prime}(t) d t d u+\int_{0}^{x} \int_{0}^{u} p_{00} y_{N}(t) d t d u \\
& -\int_{0}^{x} \int_{0}^{u} \sum_{r=0}^{N} f_{r} t^{r} d t d u+\int_{0}^{x} \int_{0}^{u}\left(\tau_{1} T_{N}(x)+\tau_{2} T_{N-1}(x)\right) d t d u
\end{aligned}
$$

Further simplification of equation (2-8), we have

$$
\begin{gathered}
p_{20} \int_{0}^{x} \int_{0}^{u} y_{N}{ }^{\prime \prime}(t) d t d u+p_{21} \iint t y_{N}{ }^{\prime \prime}(t) d t d u+p_{22} \int_{0}^{x} \int_{0}^{u} t^{2} y_{N}{ }^{\prime \prime}(t) d t d u+p_{10} \int_{0}^{x} \int_{0}^{u} y_{N}{ }^{\prime}(t) d t d u \\
+p_{11} \int_{0}^{x} \int_{0}^{u} t y_{N}{ }^{\prime}(t) d t d u+p_{10} \int_{0}^{x} \int_{0}^{u} y_{N}(t) d t d u \\
\left.+\int_{0}^{x} \int_{0}^{u} \sum_{r=0}^{N} f_{r} t^{r} d t d u+\tau_{1} \int_{0}^{x} \int_{0}^{u} T_{N}(t) d t d u+\tau_{2} \int_{0}^{x} \int_{0}^{u} T_{N}(t)\right) d t d u
\end{gathered}
$$

Hence, integrating each term one by one and making use of the given conditions, we have

$$
\begin{aligned}
& \left.p_{20} \int\left[y^{\prime}{ }_{N}(u)-\int_{0}^{u} y^{\prime}{ }_{N}(t) d t\right] d u+p_{21} \int_{0}^{x}\left[u y^{\prime}{ }_{N}(u)-\left(y_{N}(u)-y_{N}(0)\right)\right] d u\right] \\
& +p_{22} \int\left[u^{2} y^{\prime}{ }_{N}(u)-2 u y_{N}(u)+2 \int_{0}^{x} y_{N}(t) d t\right] d u+p_{10} \int_{0}^{x}\left[y_{N}(u)-y_{N}(0)\right] d u \\
& +p_{11} \int_{0}^{x}\left[u y_{N}(u)-\int_{0}^{x} y_{N}(t) d t\right] d u+p_{00} \int_{0}^{x} \int_{0}^{u} y_{N}(t) d t d u \\
& =\int_{0}^{x} \int_{0}^{u} \sum_{r=0}^{N} f_{r} t^{r} d t d u+\tau_{1} \sum_{r=0}^{N+2} C_{r}{ }^{(N+2)} x^{r}+\tau_{2} \sum_{r=0}^{N+1} C_{r}{ }^{(N+1)} x^{r}
\end{aligned}
$$


On further simplification of equation (2.10), we have

$$
\begin{aligned}
-p_{20} \alpha_{0}-p_{20} & \alpha_{1} x+p_{21} \alpha_{0} x-p_{10} \alpha_{0} x+\sum_{r=0}^{N} p_{20} q_{r} x^{r}+\sum_{r=0}^{N}\left[\frac{(r-1) p_{21}+p_{10}}{r+1}\right] a_{r} x^{r+1} \\
& +\sum_{r=0}^{N}\left[\frac{\left(r^{2}-r\right) p_{22}+r p_{11}+p_{00}}{(r+1)(r+2)}\right] a_{r} x^{r+2} \\
= & \sum_{r=0}^{N} f_{r}\left[\frac{x^{r+2}}{(r+1)(r+2)}\right]+\tau_{1} \sum_{r=0}^{N+2} C_{r}{ }^{N+2} x^{r}+\tau_{2} \sum_{r=o}^{N} C_{r}{ }^{N+1} x^{r}
\end{aligned}
$$

Thus, collecting like terms of $a_{i}(i \geq 0)$ in (2.11) and further simplification, we have

$$
\begin{aligned}
& {\left[p_{20}+\left(p_{10}-p_{21}\right) x+\frac{p_{00}}{2}\right] a_{0}+\left[p_{20} x+\frac{p_{20} x^{2}}{2}+\left(\frac{p_{11}+p_{00}}{6} x^{3}\right] a_{1}\right.} \\
& +\left[p_{20} x^{2}+\left(\frac{p_{21}+p_{10}}{3} x^{3}+\left(\frac{p_{00}+2 p_{11}+2 p_{22}}{12}\right) x^{4}\right] a_{2}\right. \\
& +\left[p_{20} x^{3}+\left(\frac{p_{10}+2 p_{21}}{4}\right) x^{4}+\left(\frac{p_{00}+3 p_{11}+6 p_{22}}{20}\right) x^{5}\right] a_{3} \\
& +\left[p_{20} x^{4}+\left(\frac{p_{10}+3 p_{22}}{5}\right) x^{5}+\left(\frac{p_{00}+4 p_{11}+12 p_{22}}{30}\right) x^{6}\right] a_{4} \\
& +\left[p_{20} x^{N}+\left(\frac{p_{10}+(N-1) p_{21}}{N+1}\right) x^{N+1}+\left(\frac{p_{00}+N p_{11}+N(N-1) p_{22}}{(\mathrm{~N}+1)(\mathrm{N}+2)}\right) x^{N+2}\right] a_{\mathrm{N}} \\
& -\tau_{1}\left\{C_{0}^{(N+2)}+C_{1}^{(N+2)} x+C_{2}^{(N+2)} x^{2}+\ldots \ldots .+C_{N+2}^{(N+2)} x^{N+2}\right\} \\
& -\tau_{2}\left(C_{0}^{(N+1)}+C_{1}^{(N+1)} x+C_{2}^{(N+1)} x^{2}+\ldots \ldots+C_{N+1}^{(N+1)} x^{N+1}\right) \\
& =\frac{x^{2}}{2} f_{0}+\frac{x^{3}}{6} f_{1}+\frac{x^{4}}{12} f_{2}+\ldots \ldots . .+\frac{x^{N+2}}{(N+1)(N+2)} f_{N} \\
& +p_{20} \alpha_{0}+p_{20} \alpha_{1} x+p_{21} \alpha_{1} x-p_{21} \alpha_{0}+p_{10} \alpha_{0} x
\end{aligned}
$$

Hence, equation (2.12) is then collocated at point $x=x_{k}$, we have

$$
\begin{aligned}
& {\left[p_{20}+\left(p_{10}-p_{21}\right) x_{k}+\frac{p_{00}}{2}\right] a_{0}+\left[p_{20} x_{k}+\frac{p_{20} x_{k}^{2}}{2}+\left(\frac{p_{11}+p_{00}}{6} x_{k}^{3}\right] a_{1}\right.} \\
& +\left[p_{20} x_{k}^{2}+\left(\frac{p_{21}+p_{10}}{3}\right) x_{k}^{3}+\left(\frac{p_{00}+2 p_{11}+2 p_{22}}{12}\right) x_{k}^{4}\right] a_{2} \\
& +\left[p_{20} x_{k}^{3}+\left(\frac{p_{10}+2 p_{21}}{4}\right) x_{k}^{4}+\left(\frac{p_{00}+3 p_{11}+6 p_{22}}{20}\right) x_{k}{ }^{5}\right] a_{3}
\end{aligned}
$$




$$
\begin{aligned}
& +\left[p_{20} x_{k}{ }^{4}+\left(\frac{p_{10}+3 p_{22}}{5}\right) x_{k}{ }^{5}+\left(\frac{p_{00}+4 p_{11}+12 p_{22}}{30}\right) x_{k}{ }^{6}\right] a_{4} \\
& \cdot \\
& +\left[p_{20} x_{k}{ }^{N}+\left(\frac{p_{10}+(N-1) p_{21}}{N+1}\right) x_{k}{ }^{N+1}+\left(\frac{p_{00}+N p_{11}+N(N-1) p_{22}}{(\mathrm{~N}+1)(\mathrm{N}+2)} x_{k}{ }^{N+2}\right] a_{\mathrm{N}}\right. \\
& -\tau_{1}\left\{C_{0}^{(N+2)}+C_{1}^{(N+2)} x_{k}+C_{2}^{(N+2)} x_{k}{ }^{2}+\ldots \ldots . .+C_{N+2}^{(N+2)} x_{k}{ }^{N+2}\right\} \\
& -\tau_{2}\left(C_{0}^{(N+1)}+C_{1}^{(N+1)} x_{k}+C_{2}^{(N+1)} x_{k}{ }^{2}+\ldots . .+C_{N+1}^{(N+1)} x_{k}{ }^{N+1}\right) \\
& =\frac{x_{k}{ }^{2}}{2} f_{0}+\frac{x_{k}{ }^{3}}{6} f_{1}+\frac{x_{k}{ }^{4}}{12} f_{2}+\ldots \ldots . .+\frac{x_{k}{ }^{N+2}}{(N+1)(N+2)} f_{N} \\
& \quad+p_{20} \alpha_{0}+p_{20} \alpha_{1} x_{k}+p_{21} \alpha_{1} x_{k}-p_{21} \alpha_{0}+p_{10} \alpha_{0} x_{k}
\end{aligned}
$$

Where,

$$
x_{k}=a+\frac{(b-a) k}{N+2} ; k=1,2,3,---, N+1
$$

Thus, equation (2.13) gives $(\mathrm{N}+1)$ algebraic linear system of equations in $(\mathrm{N}+3)$ unknown constants. Two extra equations are obtained using equations (2.2) and (2. 3). Altogether, we have $(\mathrm{N}+3)$ algebraic linear system of equations which are then put in matrix form as follows:

$$
A x=B
$$

The algebraic linear system of equations are the solved by Gaussian elimination method to obtain the unknown constants which are the substituted into the approximate solution.

\section{Numerical Examples}

In this section, we solved the following linear and nonlinear second order ordinary differential equations by Perturbed Integral Collocation Approximation method for different values of $\mathrm{N}$ ( the degree of approximant)

Example 1

Solve the following boundary value problem

$$
y^{\prime \prime}(x)+(x+1) y^{\prime}(x)-2 y(x)=\left(1-x^{2}\right) e^{-x}, 0 \leq x \leq 1
$$

The conditions are given as

$$
y(0)=-1 \text { and } y(1)=0 \text {. The exact solution is } y(x)=(x-1) e^{-x}
$$

Table 1: Results obtained for case $\mathrm{N}=4$

\begin{tabular}{|l|l|l|l|}
\hline $\mathrm{X}$ & Exact $\mathrm{y}(\mathrm{x})$ & ${\text { Approximation } \mathrm{y}_{N}(\mathrm{x})}$ & ${\text { Error }=\mathrm{y}(\mathrm{x})-\mathrm{y}_{N}(\mathrm{x})}$ \\
\hline 0.1 & 0.994653826 & 0.994650184 & $3.642 \times 10^{-6}$ \\
\hline 0.2 & 0.977122206 & 0.977113687 & $8.519 \times 10^{-6}$ \\
\hline 0.3 & 0.944901165 & 0.944837925 & $6.324 \times 10^{-5}$ \\
\hline 0,4 & 0.895094818 & 0.895044428 & $5.039 \times 10^{-5}$ \\
\hline 0.5 & 0.824360635 & 0.824311665 & $4.897 \times 10^{-5}$ \\
\hline 0.6 & 0.728847520 & 0.728801420 & $4.610 \times 10^{-5}$ \\
\hline 0.7 & 0.604125812 & 0.604085862 & $3.995 \times 10^{-5}$ \\
\hline 0.8 & 0.445108185 & 0.445070925 & $3.726 \times 10^{-5}$ \\
\hline 0.9 & 0.245960311 & 0.245588911 & $3.714 \times 10^{-5}$ \\
\hline 1.0 & 0 & 0.000000005 & $5.000 \times 10^{-8}$ \\
\hline
\end{tabular}


Table 2: Results obtained for case $\mathrm{N}=8$

\begin{tabular}{|l|l|l|l|}
\hline $\mathrm{x}$ & Exact $\mathrm{y}(\mathrm{x})$ & Approximation $\mathrm{y}_{N}(\mathrm{x})$ & Error $=\mathrm{y}(\mathrm{x})-\mathrm{y}_{N}(\mathrm{x})$ \\
\hline 0.1 & 0.994653826 & 0.994653816 & $9.813 \times 10^{-8}$ \\
\hline 0.2 & 0.977122206 & 0.977122197 & $9.003 \times 10^{-8}$ \\
\hline 0.3 & 0.944901165 & 0.944901080 & $8.443 \times 10^{-8}$ \\
\hline 0.4 & 0.895094818 & 0.895094738 & $8.002 \times 10^{-8}$ \\
\hline 0.5 & 0.824360635 & 0.824360567 & $6.792 \times 10^{-8}$ \\
\hline 0.6 & 0.728847520 & 0.7288474456 & $6.387 \times 10^{-8}$ \\
\hline 0.7 & 0.604125812 & 0.604125752 & $5.996 \times 10^{-8}$ \\
\hline 0.8 & 0.445108185 & 0.445108138 & $4.772 \times 10^{-8}$ \\
\hline 0.9 & 0.245960311 & 0.245960264 & $4.678 \times 10^{-8}$ \\
\hline 1.0 & 0 & 0.000000002 & $2.000 \times 10^{-9}$ \\
\hline
\end{tabular}

Example 2

Solve the following boundary value problem

$$
y^{\prime \prime}(x)+e^{x} y^{\prime}(x)-x y(x)=e^{-x}\left(-x^{2}+2 x-, 0 \leq x \leq 1\right.
$$

The conditions are given as $y(0)=-1$ and $y(1)=0$

The exact solution is $y(x)=(x-1) e^{-x}$

Table : Results obtained for case $\mathrm{N}=4$

\begin{tabular}{|l|l|l|l|}
\hline $\mathrm{x}$ & Exact $\mathrm{y}(\mathrm{x})$ & Approximation $\mathrm{y}_{N}(\mathrm{x})$ & Error $=\mid \mathrm{y}(\mathrm{x})-\mathrm{y}_{N}(\mathrm{x})$ \\
\hline 0.1 & 0.994653826 & 0.994654257 & $4.318 \times 10^{-7}$ \\
\hline 0.2 & 0.977122206 & 0.977149086 & $2.688 \times 10^{-5}$ \\
\hline 0. & 0.944901165 & 0.945862265 & $9.611 \times 10^{-4}$ \\
\hline 0.4 & 0.895094818 & 0.895828678 & $7.338 \times 10^{-4}$ \\
\hline 0.5 & 0.824360635 & 0.825061235 & $7.006 \times 10^{-4}$ \\
\hline 0.6 & 0.728847520 & 0.729310720 & $4.632 \times 10^{-4}$ \\
\hline 0.7 & 0.604125812 & 0.604548712 & $4.229 \times 10^{-4}$ \\
\hline 0.8 & 0.445108185 & 0.445419385 & $3.112 \times 10^{-4}$ \\
\hline 0.9 & 0.245960311 & 0.246253011 & $2.927 \times 10^{-4}$ \\
\hline 1.0 & 0 & 0.000083430 & $8.343 \times 10^{-5}$ \\
\hline
\end{tabular}

Table 4: Results obtained for case $\mathrm{N}=8$

\begin{tabular}{|l|l|l|l|}
\hline $\mathrm{x}$ & Exact $\mathrm{y}(\mathrm{x})$ & Approximation $\mathrm{y}_{N}(\mathrm{x})$ & Error= $\mid \mathrm{y}(\mathrm{x})-\mathrm{y}_{N}(\mathrm{x})$ \\
\hline 0.1 & 0.994653826 & 0.994654464 & $6.389 \times 10^{-7}$ \\
\hline 0.2 & 0.977122206 & 0.977122833 & $6.271 \times 10^{-7}$ \\
\hline 0.3 & 0.944901165 & 0.944901743 & $5.789 \times 10^{-7}$ \\
\hline 0.4 & 0.895094818 & 0.895099551 & $4.733 \times 10^{-7}$ \\
\hline 0.5 & 0.824360635 & 0.824361096 & $4.613 \times 10^{-7}$ \\
\hline 0.6 & 0.728847520 & 0.728847951 & $4.319 \times 10^{-7}$ \\
\hline 0.7 & 0.604125812 & 0.604126234 & $4.227 \times 10^{-7}$ \\
\hline 0.8 & 0.445108185 & 0.445109269 & $4.197 \times 10^{-7}$ \\
\hline 0.9 & 0.245960311 & 0.245960711 & $4.007 \times 10^{-7}$ \\
\hline 1.0 & 0 & 0.0000002167 & $2.167 \times 10^{-7}$ \\
\hline
\end{tabular}


Example 3

Consider a second order differential equation
$(1-x) y^{\prime \prime}(x)-x y^{\prime}(x)=2$,
$0 \leq x \leq 1$

Together with the boundary conditions $y(0)=1$ and $y^{\prime}(0)=0$

The exact solution is given as $y(x)=1+\left(\operatorname{Sin}^{-1} x\right)^{2}$

Table 5: Results obtained for case $\mathrm{N}=4$

\begin{tabular}{|l|l|l|l|}
\hline $\mathrm{x}$ & Exact $\mathrm{y}(\mathrm{x})$ & Approximation $\mathrm{y}_{N}(\mathrm{x})$ & $\begin{array}{l}\text { Error }=\mid \mathrm{y}(\mathrm{x})-_{N}(\mathrm{x}) \\
\mathrm{y}_{N}\end{array}$ \\
\hline 0.1 & 1.0100335120 & 1.0093225460 & $7.110 \times 10^{-4}$ \\
\hline 0.2 & 1.0405450120 & 1.0394037450 & $1.508 \times 10^{-4}$ \\
\hline 0.3 & 1.0928376130 & 1.0926421740 & $1.954 \times 10^{-4}$ \\
\hline 0.4 & 1.1693461150 & 1.1688525770 & $4.935 \times 10^{-4}$ \\
\hline 0.5 & 1.2741556780 & 1.2739727640 & $1.829 \times 10^{-4}$ \\
\hline 0.6 & 1.4140936770 & 1.4137056980 & $3.880 \times 10^{-4}$ \\
\hline 0.7 & 1.6012412780 & 1.6055128000 & $4.139 \times 10^{-4}$ \\
\hline 0.8 & 1.8598764210 & 1.8593445900 & $5.318 \times 10^{-4}$ \\
\hline 0.9 & 2.2538837670 & 2.2532296100 & $6.542 \times 10^{-4}$ \\
\hline 1.0 & 3.4674011000 & 3.4674073580 & $6.258 \times 10^{-6}$ \\
\hline
\end{tabular}

Table 6: Results obtained for case $\mathrm{N}=8$

\begin{tabular}{|l|l|l|l|}
\hline $\mathrm{x}$ & Exact $\mathrm{y}(\mathrm{x})$ & Approximation $_{N}(\mathrm{x})$ & ${\text { Error }=\mid \mathrm{y}(\mathrm{x})-\mathrm{y}_{N}(\mathrm{x})}$ \\
\hline 0.1 & 1.0100335120 & 1.010335111 & $9.128 \times 10^{-9}$ \\
\hline 0.2 & 1.0405450120 & 1.045450066 & $5.412 \times 10^{-8}$ \\
\hline 0.3 & 1.0928376130 & 1.092837346 & $2.671 \times 10^{-7}$ \\
\hline 0.4 & 1.1693461150 & 1.169345773 & $3.416 \times 10^{-7}$ \\
\hline 0.5 & 1.2741556780 & 1,274155280 & $3.978 \times 10^{-7}$ \\
\hline 0.6 & 1.4140936770 & 1.414093153 & $5.241 \times 10^{-7}$ \\
\hline 0.7 & 1.6012412780 & 1.691240699 & $5.786 \times 10^{-7}$ \\
\hline 0.8 & 1.8598764210 & 1.858875742 & $6.789 \times 10^{-7}$ \\
\hline 0.9 & 2.2538837670 & 2.253836909 & $7.613 \times 10^{-7}$ \\
\hline 1.0 & 3.4674011000 & 3.467401091 & $9.42110^{-9}$ \\
\hline
\end{tabular}

\section{Discussion of Results}

The numerical results show that Perturbed integral collocation method is a promising and powerful tool for solving second order ordinary differential equations with variable coefficients which many numerical methods could not solve directly because over determination. Also, the results are in total agreement with the analytical solution as the degree of the approximant $(\mathrm{N})$ increases.

\section{References}

[1] Fox, L. (1962) . Numerical Solution of Ordinary Differential Equations and Partial Differential Equations. Pergamum, Oxford.

[2] Gerald, D. (2005). Numerical Methods in Engineering and Sciences. $7^{\text {th }}$ ed., Kanna Publishers, Delhi.

[3] Hosseini Allahadi M. (2005). Solving Ordinary Differential Equation using the Perturbation term of the Tau Method over SemiInfinite Intervals. Far East J. Appli. Math. 4(), 295 - 303

[4] Taiwo, O . A and Olagunju, A. S. (2006). Perturbed Segmented Domain Collocation Tau Method for the Numerical Solution of Second Order Boundary Value Problems. J. of NAMP, 10, 293 - 298

[5] Taiwo, O . A. and Olagunju, A. S. (2011). Chebyshev Methods for the Numerical Solution of $4^{\text {th }}$ Order Differential Equations. Pioneer J. of Mathematics and Mathematical Sciences. 3(1), $73-82$. 